\title{
Gemini VRI data of counterparts associated to X-ray sources in CMa R1
}

\author{
J. Gregorio-Hetem ${ }^{1}$, C. V. Rodrigues ${ }^{2}$ and T. Montmerle ${ }^{3}$ \\ ${ }^{1}$ Universidade de São Paulo, Brazil, email: jane@astro.iag.usp.br \\ ${ }^{2}$ INPE, Brazil, email: claudiavr@das.inpe.br \\ ${ }^{3}$ Université de Grenoble, France, email: montmerle@obs.ujf-grenoble.fr
}

\begin{abstract}
The molecular cloud Canis Major R1 (CMa R1) contains several embedded stellar clusters associated to a ring of nebular emission, which is an expanding shell suggested to be a supernova remnant (SNR) inducing the star formation in this region (Herbst \& Assousa 1977, Comerón et al. 1998). However, there are alternatives to the SNR hypothesis, since the shell-like structure could be produced by strong stellar winds or an evolving HII region, as suggested by Reynolds \& Ogden (1978), Blitz (1980), and Pyatunina \& Taraskin (1986), for example. Two main challenges have motivated us to investigate this interesting region: (i) to conduct a stellar population study, from 7 to 0.4 solar masses, and (ii) to verify the evolutionary status of embedded cluster members. This contribution is dedicated to report VRI data obtained with Gemini South telescope in the direction of six X-ray sources that are probably unresolved. The results reveal several faint candidates that could be multiple counterparts of X-ray emitters detected by ROSAT as single sources (Gregorio-Hetem, Montmerle \& Marciotto 2003). These fields have not been observed in more recent X-ray surveys. The V-R and R-I colours were estimated for the objects associated with the position of the $\mathrm{X}$-ray emission, aiming to distinguish between field stars and members of the cloud. For each ROSAT source, it has been detected the following number of candidates, which we suggest to be stellar groups: src15 has 7 possible optical counterparts ( $86 \%$ of them are NIR sources); src17 has 14 counterparts ( $71 \%$ are NIR sources); src37 has 11 (73\% NIR); src42 has 16 (56\% NIR); src44 has 10 (80\% NIR); and src55 has $6(67 \%$ NIR). Investigating the evolutionary scenario of the embedded stellar clusters associated to X-ray emitters, which are probably very young, is a unique opportunity to better understand the star formation process in CMa R1 and to test SNR models, verifying the hypothesis of induced star formation in this region.
\end{abstract}
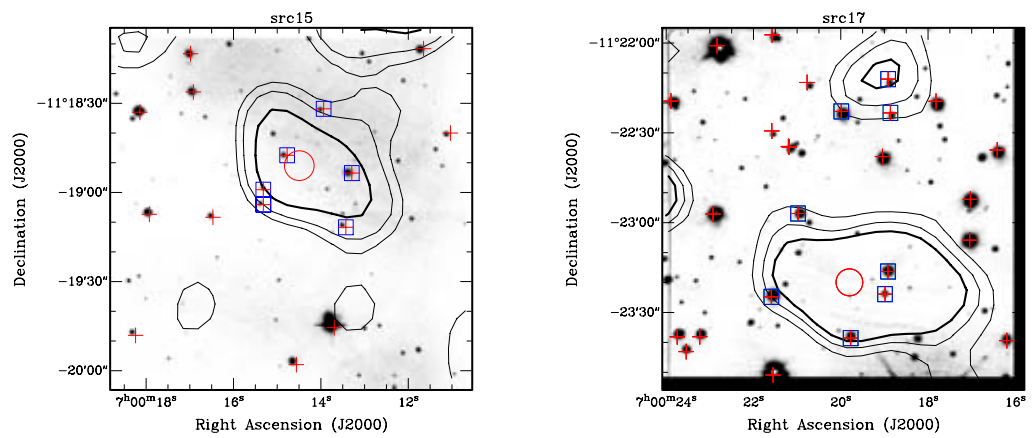

Figure 1. Optical image showing the contours of the X-Ray emission in the fields src15 and src17. The 2MASS catalogue was inspected searching for near-infrared (NIR) counterparts (crosses) related to the optical candidates (squares). Colour-magnitude diagrams have been constructed to evaluate the evolutionary status of the stellar groups.

Keywords. ISM: CMa clouds, Stars: pre-main sequence, X-rays: stars 